\title{
Chromatic-Luminance and True-Texture Analysis for Image Mining in the Social Sciences
}

\author{
Benjamin Lucas ${ }^{1}$ and Ben Kozary ${ }^{2}$ \\ ${ }^{1}$ Data Driven Discovery Initiative (3Di), University of Nottingham, United Kingdom \\ ${ }^{2}$ Facebook Inc., United States
}

March 2021

\begin{abstract}
This research note highlights the use of raw HSV (Hue, Saturation, Value) colorspace representation (capturing chromatic-luminance), and true-texture (matrix-based) representation of images for image mining applications in the social sciences. Specifically: we focus here on the basics of teaching computers to 'think like people' in making decisions about what visual content is most interesting or important to human viewers. Our examples capture the facts that, (a) computers see 'colors as numbers', rather than as meaningful sections of an image, and (b) computers see texture as 'numbers', rather than as meaningful 'hard' or 'soft' sections of an image. Illustrations are provided using the R packages 'colorfindr', 'glcm', 'imager' and 'plotly'.
\end{abstract}

\section{Introduction}

Marketing and strategic communications experts are fascinated with the goal of engineering multimedia communication to generate engagement and virality online (Berger and Milkman, 2012; Akpinar and Berger, 2017). In the case of visual content, beyond carefully timed campaign scheduling, this engineering involves: (a) the calculated combination and layout of perceptual design features such as morphology, geometry, texture, color and luminance, (b) their lower-level (pixel-level) building blocks (see: Pieters et al., 2010; Sample et al., 2019), and (c) balancing information represented as text (e.g. product description, product specification, prices) alongside visual imagery (see: Townsend and Kahn, 2014 for an extended discussion). Related research dives deeper into visual semantic interpretation, focusing in particular on high-level features such as human presence (Bakhshi et al., 2014; Berger and Barasch, 2018; Bruno et al., 2019; McDuff and Berger, 2019; Hartmann et al., 2020), given the broad potential of such features to be engineered to shape viewer reactions. 
These basic design considerations (i.e. perceptual image features, text-versusimagery balance, and conceptual semantic interpretation), and the benefits of quantifying them in an efficient manner for empirical research purposes, apply whether the 'product' is for-profit or not-for-profit. Put simply, when it comes to driving visual engagement and virality, the importance of calculated and measurable design is consistent, regardless of whether the visual message concerns a commercialized product or service, on the one hand; or, on the other, an activist's message, a social movement, or political call-to-action, as highlighted by the rise of interest in political communication and social mobilization in online communities (e.g. Fisher, 2019; Xi et al., 2020).

Recent research in the marketing and consumer psychology literatures also points toward a need to understand visual data in a more comprehensive manner, to support the answering of new research questions around individual visual perception and preferences within the broader fields of consumer perception and sensory marketing (e.g. Matz et al., 2019; Sample et al., 2019). This need is especially pertinent in the exploration of haptics in marketing communications (Peck and Childers, 2003; Jha et al., 2020).

Broadly, all visual engineering for strategic communications purposes is anchored in clutter cut-through (Pieters et al., 2007; Rosenholtz et al., 2007), the enablement of processing fluency (Shapiro, 1999; Lee and Labroo, 2004), and salience and recall maximization (Alba and Chattopadhyay, 1986; Ambler et al., 2004; Yantis, 2005). To begin to understand such processes in a reliable manner that reflects actual human perception thus requires the following: (a) the measurement of color and luminance based on holistic and data-driven approaches for combined "chromatic-luminance" extraction and analysis (see: Pridmore 2009), and, (b) true-texture extraction and analysis (mentioned in: Balducci and Marinova, 2018) versus 'textural' feature extraction and analysis.

In summary: we are focused here on colors, lighting and texture - specifically, the way in which these shape how humans perceive images, and the basics of teaching computers to 'think like people' in making decisions about what visual content is most interesting and / or important for human viewers (based on the concept of dividing digital images into components that 'real people' can understand). Our examples capture the facts that (a) computers see 'colors as numbers', rather than as meaningful sections of an image, and (b) computers see texture in images as 'numbers', rather than as meaningful 'hard' or 'soft' sections of an image.

\section{Chromatic-Luminance}

The effect of color perception has been widely explored in the marketing and consumer psychology literatures (e.g. Deng et al., 2010; Labrecque and Milne, 2012; Kareklas et al., 2014; Bakhshi et al., 2015; Hagtvedt and Brasel, 2017; 
Hagtvedt, 2019), as well as within the broader psychology and neuroscience literatures (Xian and Shevell, 2004; Holmes and Regier, 2017; Jonauskaite et al., 2019). Color alone is also an important basis for determining regions-of-interest (ROIs) (Lou et al., 2014), and thus has critical implications for understanding selective perception, and perceptual grouping (Xian and Shevell, 2004) - that being the way in which a viewer of visual stimuli quickly summarizes patterns present within that stimuli to create meaning.

The HSV colorspace (Hue, Saturation, Value) is regarded as an appropriate representation of human color perception (see: Schwarz, Cowan and Beatty, 1987; Bakhshi and Gilbert, 2015). A practical example of how ROIs can be determined via the HSV colorspace is illustrated in Figure 1, and was generated using the R package 'colorfindr' (Zumbach, 2019). Figure 1 shows an image with dominant red, green and blue (RGB) dimensions, as well as what this image looks like when decomposed into HSV colorspace representation.
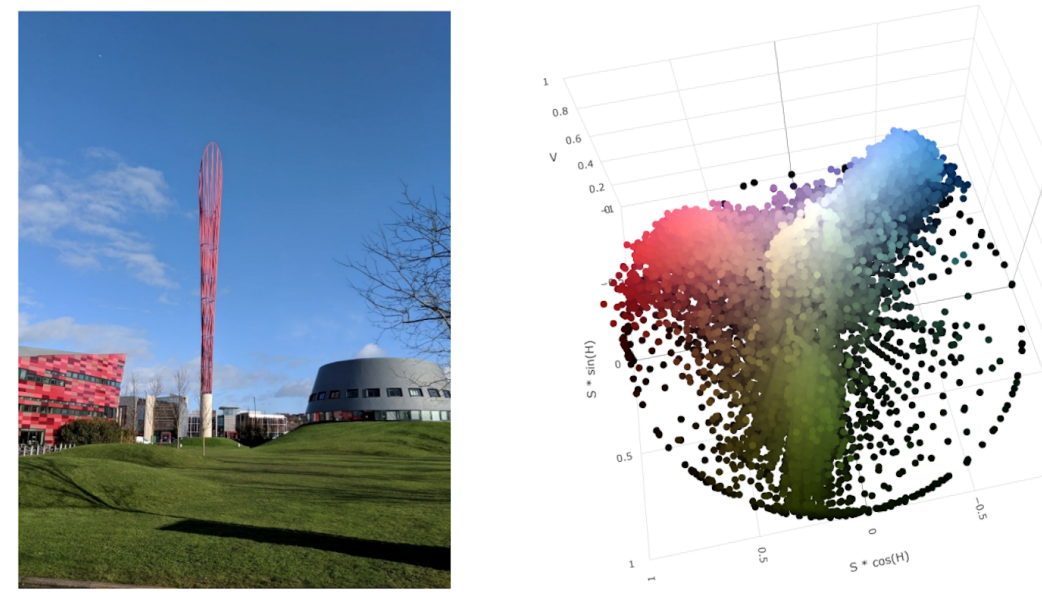

Figure 1: HSV Colorspace Example

Figures 2a and 3a further illustrate this concept at different levels of extraction 'resolution' (i.e. number of color-points extracted), with prominent background and foreground colors dominant, and subtler features (the Beagle's collar in Figure 2a, and the Beagle's ball in Figure 3a) noticeably emergent.

Conversely, Figures $2 \mathrm{~b}$ and $3 \mathrm{~b}$ show a simple example of color summarization (given an image with complex gradients, as is the case with photographic images) - in this case based on the top five most prevalent colors used at the pixel level (and represented as hexadecimal color palettes). The left panel of Figure $2 \mathrm{~b}$ shows that, following this simple color summarization approach, the original image is treated as largely black and white. Meanwhile, the right panel of Figure $2 \mathrm{~b}$ illustrates that, when using an arbitrary bounding box highlighting 
the Beagle's collar (e.g. versus a data-driven clustering approach), a noticeable amount of color micro-variation exists (i.e. beyond human perceptual grouping). Furthermore, Figure 3b shows that the original image (left panel) is treated as predominantly green (with a large percentage of dark gray), whereas an arbitrary bounding box highlighting the Beagle's ball (right panel) shows perceptible color micro-variation.

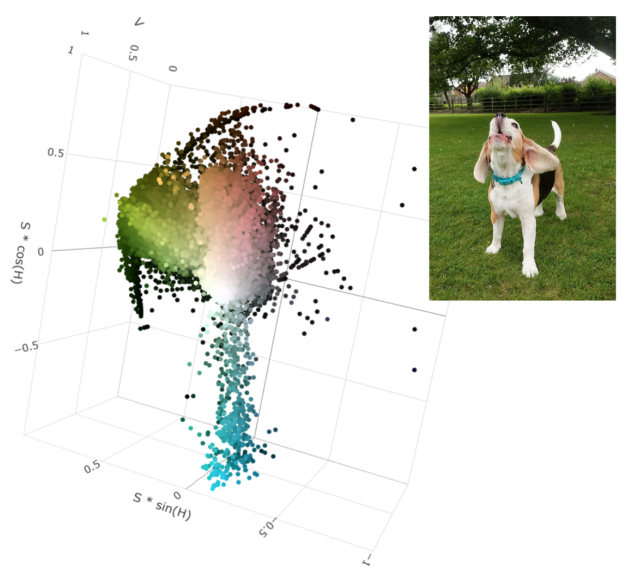

Figure 2a: Beagle in HSV Colorspace (1)
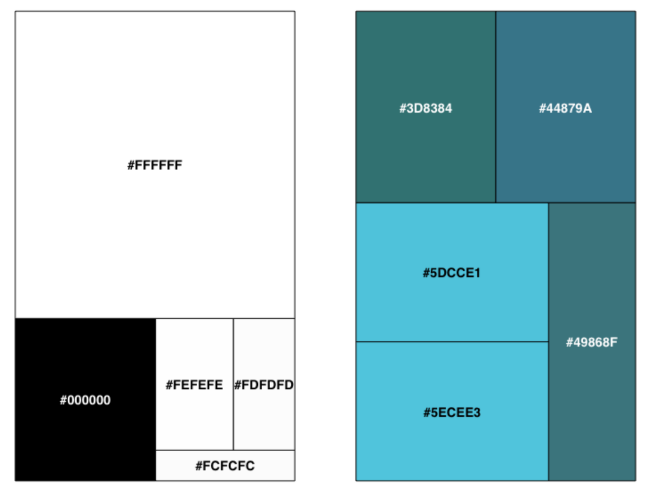

Figure 2b: Hexadecimal Comparison (1) (L: Original, R: ROI) 


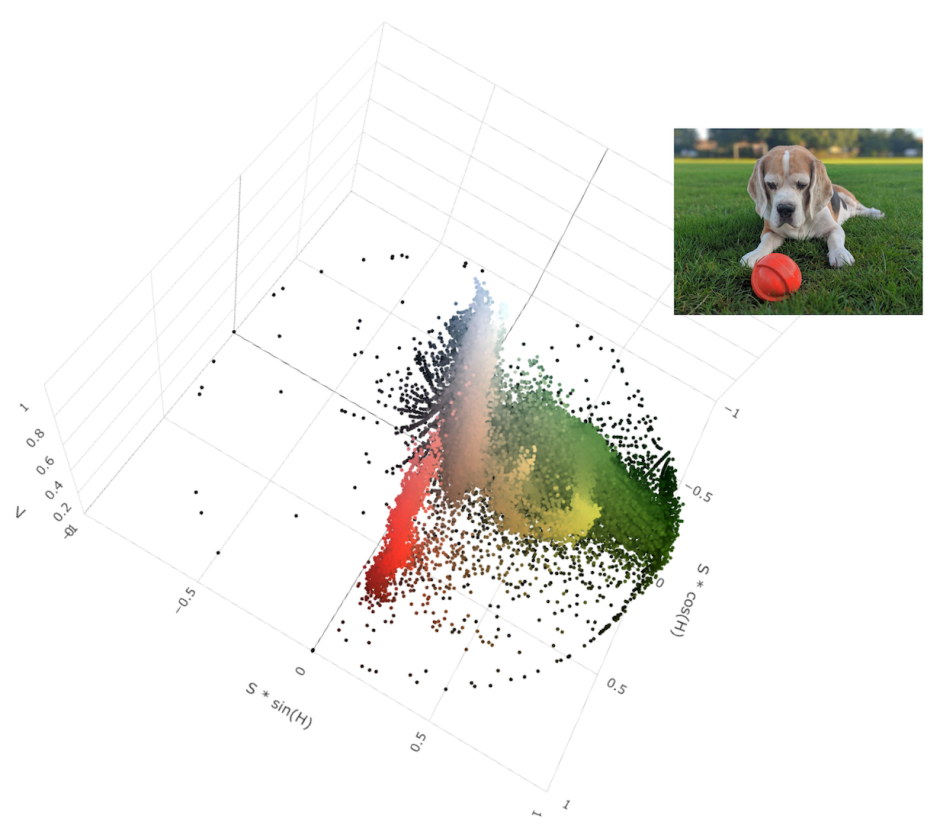

Figure 3a: Beagle in HSV Colorspace (2)
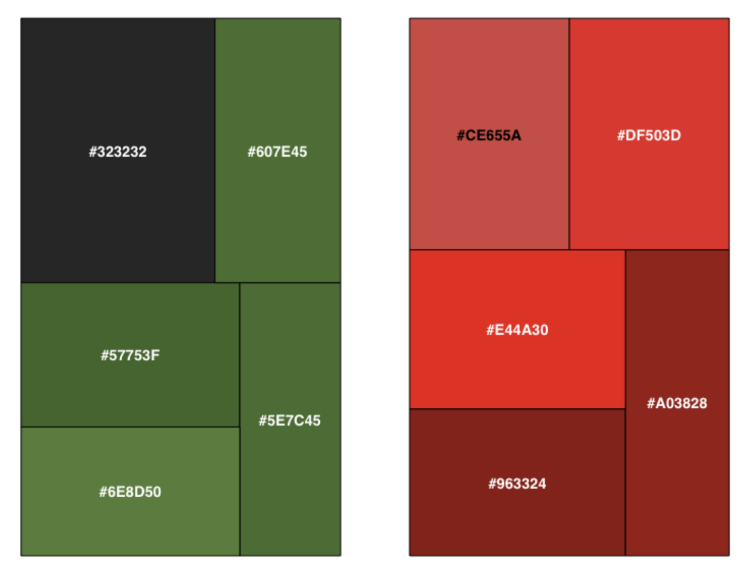

Figure 3b: Hexadecimal Comparison (2) (L: Original, R: ROI) 
The main point here is that by taking a data-driven approach to color feature extraction (e.g. using clustering approaches - as illustrated in Figures 2a and 3a), much more useful and detailed information about prominent color groupings can be captured (relative to a simple color summarization approach - as in Figures $2 \mathrm{~b}$ and $3 \mathrm{~b}$ ). This has substantial implications for scientific and industrial image mining, especially along the lines of reducing bias in training sets and engineering features for supervised machine learning. The use of tractable abstraction methods (see for example: Gerstner et al., 2013) as a key data processing step, should thus be more greatly underscored in the social science image mining literature.

\section{Textural Features and True Texture}

Gray-level co-occurrence matrices (GLCM) are quantized representations of images used as a foundation to calculate a variety of 'textural' image features based on aggregated pixel intensities (Jacobs et al., 2016; Löfstedt et al., 2019; Matz et al., 2019). Figure 4 shows low-level 2D textural features using the $\mathrm{R}$ package 'glcm' (Zvoleff, 2019) based on this image. The most basic examples - mean (top panel) and variance (bottom panel) - are shown here.

In contrast, an approach to true-texture provides a 3D approximation based only on 2D grayscale pixel intensities. Figure 5 shows a relief plot generated using the R package 'plotly' (Sievert et al. 2019) to provide a true-texture abstraction / representation of the Beagle's face as a region-of-interest (ROI). The input was generated using the R package 'imager' (Barthelme et al., 2019), where matrix elements represent gray-level pixel intensities, and where $x$ and $y$ represent the $2 \mathrm{D}$ borders of the image, and $z$ represents depth. 

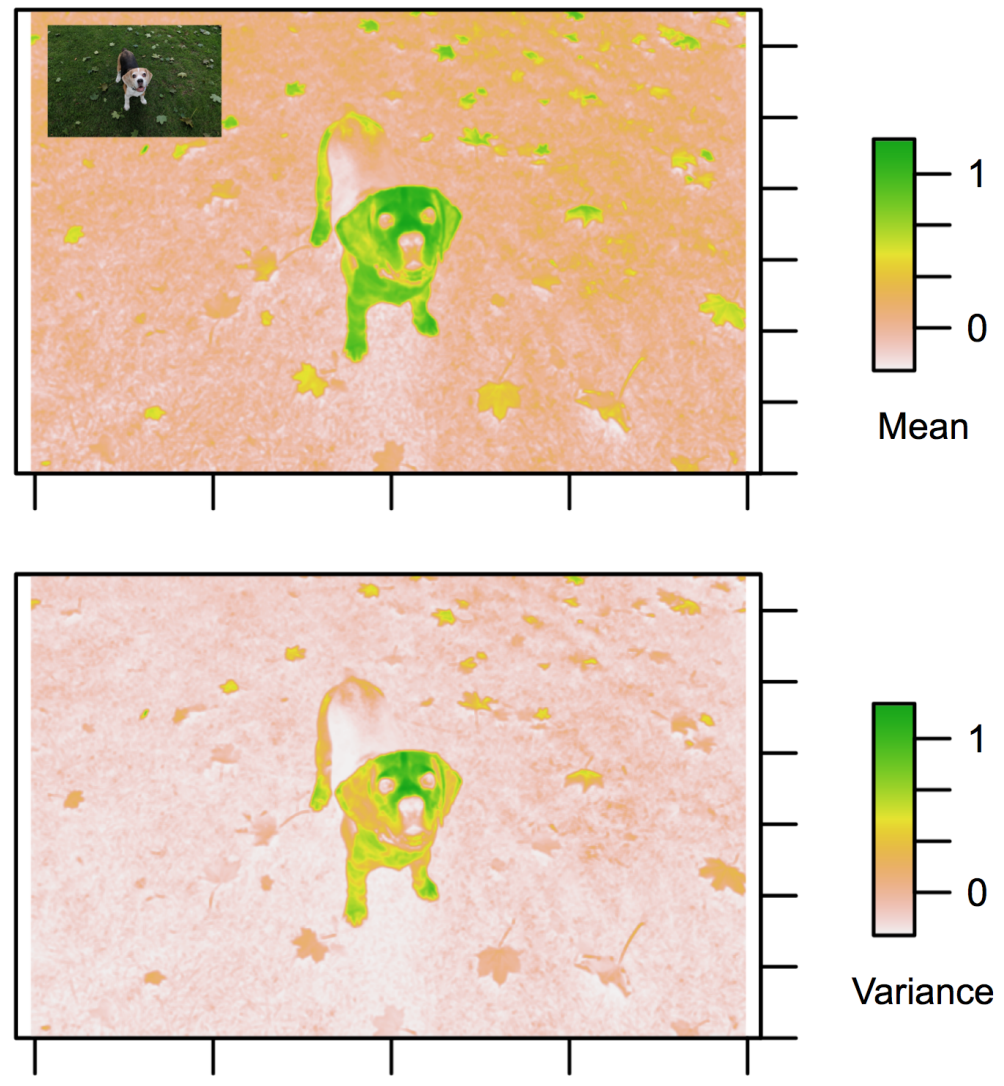

Figure 4: Visualization of Mean and Variance Textural Features

The main point here is that although low-level 'textural' features provide useful summarization of the arrangement of pixels within 2D images (e.g. contrast and entropy and their directionality - see: Brynolfsson et al., 2017), treating images first as 'raw' matrices allows for more comprehensive data-driven analysis by quantifying and identifying true-texture (e.g. hard, soft) where needed. Similarly, data-driven approaches focused on true-texture provide better information for feature engineering (i.e. aligned with human perception). This relates specifically to true-texture profiling as well as ROI comparison - even in lower-resolution images (e.g. by comparing regional uniformity - noting sections of the Beagle's face versus the grass background in Figure 5). The cost of not doing this is leaving feature selection at the mercy of biased, opaque, or insufficiently tractable methods of definition, thus resulting in models which reflect aggressively simplified quantization, rather than actual human perception. 


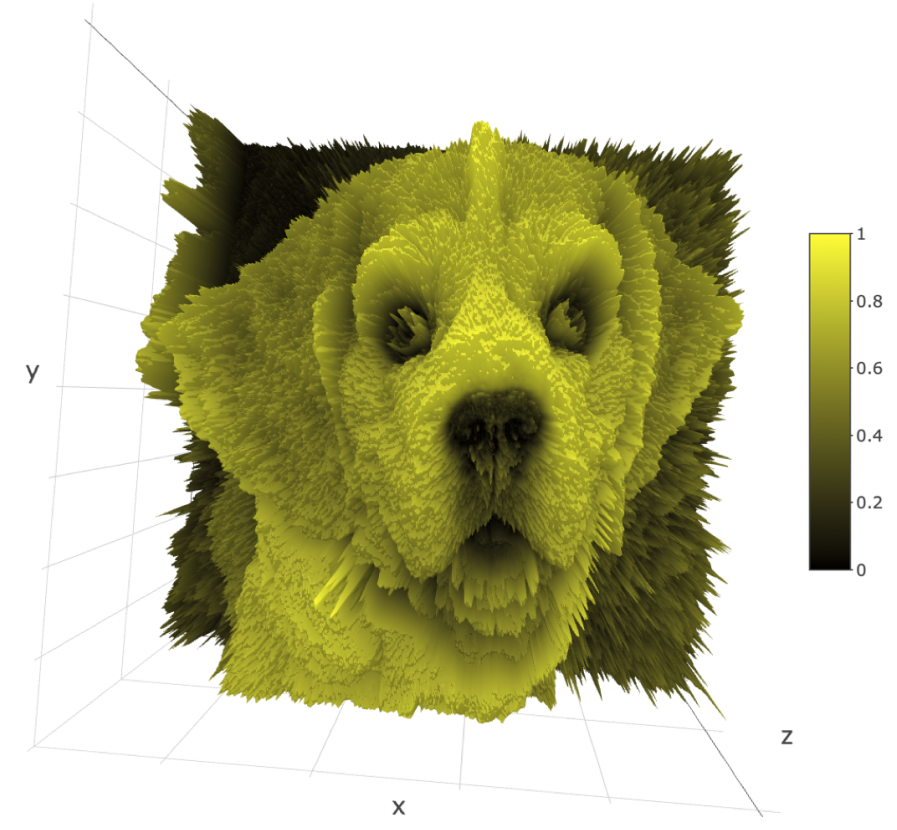

Figure 5: Visualization of 3D True-Texture

\section{Concluding Remarks}

In social science fields such as marketing research, political science, cultural studies and environmental psychology, where visual communication is widely explored - be that in the study of advertising and strategic communications, political messaging, the study of cultural artefacts and art, or the study of any given surroundings of human experience (e.g. architecture and the built environment) - there is a shared interest in streamlining the process of feature extraction from visual data, and importantly, in building deeper understanding around visual communication and aesthetics in general (see for example: Lyons, 2017; Ibarra et al., 2017; Joo and Steinert-Threlkeld, 2018; Steinert-Threlkeld, 2019; Al-Halah and Grauman, 2020; Xi et al., 2020). This divides broadly into understanding individual perceptions and preferences (Stamps, 2002; Witzel et al., 2017), as well as building generalizations about desirable and compelling image composition - a theme currently explored across the social science and computer science literatures (Badea et al., 2018; Constantin et al., 2019; Matz et al., 2019).

This research note aims to highlight that scalability should not be an overwhelming factor in methodology design in image mining in the social sciences, especially at the expense of detail and nuance. Whilst summarizations of images aid in the construction of generalizable insights across large visual corpora, 
researchers should also consider the deep potential of intra-image (versus interimage) analyses (and feature engineering) as a basis for gaining data-driven perspectives on appealing and engaging visual content. To recap and summarize, this has important implications for reducing bias in training sets and engineering features for supervised machine learning, as well as for other social science approaches, such as where data-driven quantification and quantization of image features is used as a basis for controlled experiments at scale.

\section{References}

Akpinar, E. and Berger, J., 2017. Valuable virality. Journal of Marketing Research, 54(2), pp.318-330.

Alba, J.W. and Chattopadhyay, A., 1986. Salience effects in brand recall. Journal of Marketing Research, 23(4), pp.363-369.

Al-Halah, Z. and Grauman, K., 2020. From Paris to Berlin: Discovering Fashion Style Influences Around the World. In Proceedings of the IEEE/CVF Conference on Computer Vision and Pattern Recognition (pp. 10136-10145).

Ambler, T., Braeutigam, S., Stins, J., Rose, S. and Swithenby, S., 2004. Salience and choice: neural correlates of shopping decisions. Psychology Marketing, 21(4), pp.247-261.

Badea, M., Florea, C., Florea, L. and Vertan, C., 2018. Can we teach computers to understand art? Domain adaptation for enhancing deep networks capacity to de-abstract art. Image and Vision Computing, 77, pp.21-32.

Bakhshi, S. and Gilbert, E., 2015. Red, purple and pink: The colors of diffusion on Pinterest. PloS One, 10(2), p.e0117148.

Bakhshi, S., Shamma, D.A. and Gilbert, E., 2014, April. Faces engage us: Photos with faces attract more likes and comments on instagram. In Proceedings of the SIGCHI Conference on Human Factors in Computing Systems (pp. 965-974).

Balducci, B. and Marinova, D., 2018. Unstructured data in marketing. Journal of the Academy of Marketing Science, 46(4), pp.557-590.

Barthelme, S., Tschumperle, D., Wijffels, J. and Assemlal H-E. (2019). R Package 'imager'. [Link]

Berger, J. and Barasch, A., 2018. A Candid Advantage? The Social Benefits of Candid Photos. Social Psychological and Personality Science, 9(8), pp.1010-1016.

Berger, J. and Milkman, K.L., 2012. What makes online content viral?. Journal of Marketing Research, 49(2), pp.192-

Bruno, N., Bertamini, M. and Tyler, C.W., 2019. Eye centring in selfies posted on Instagram. PloS One, 14(7), p.e0218663.

Brynolfsson, P., Nilsson, D., Torheim, T., Asklund, T., Karlsson, C.T., Trygg, J., Nyholm, T. and Garpebring, A., 2017. Haralick texture features from apparent diffusion coefficient (ADC) MRI images depend on imaging and pre-processing parameters. Scientific Reports, $7(1)$, pp.1-11.

Constantin, M.G., Redi, M., Zen, G. and Ionescu, B., 2019. Computational understanding of visual interestingness beyond semantics: literature survey and analysis of covariates. ACM Computing Surveys (CSUR), $52(2)$, p. 25.

Deng, X., Hui, S.K. and Hutchinson, J.W., 2010. Consumer preferences for color combinations: An empirical analysis of similarity-based color relationships. Journal of Consumer Psychology, 20(4), pp.476-484.

Fisher, D.R., 2019. The broader importance of \#FridaysForFuture. Nature Climate Change, 9(6), pp.430-431.

Gerstner, T., DeCarlo, D., Alexa, M., Finkelstein, A., Gingold, Y. and Nealen, A., 2013. Pixelated image abstraction with integrated user constraints. Computers Graphics, 37(5), pp.333-347.

Hagtvedt, H. and Brasel, S.A., 2017. Color saturation increases perceived product size. Journal of Consumer Research, 44(2), pp.396-413.

Hagtvedt, H., 2019. Dark is durable, light is user-friendly: The impact of color lightness on two product attribute judgments. Psychology \&s Marketing.

Hartmann, J., Heitmann, M., Schamp, C. and Netzer, O., 2019. The power of brand selfies in consumer-generated brand images. Columbia Business School Research Paper.

Holmes, K.J. and Regier, T., 2017. Categorical perception beyond the basic level: The case of warm and cool colors. Cognitive Science, 41(4), pp.1135-1147.

Ibarra, F.F., Kardan, O., Hunter, M.R., Kotabe, H.P., Meyer, F.A. and Berman, M.G., 2017. Image feature types and their predictions of aesthetic preference and naturalness. Frontiers in Psychology, 8, p.632.

Jacobs, R.H., Haak, K.V., Thumfart, S., Renken, R., Henson, B. and Cornelissen, F.W., 2016. Aesthetics by numbers: links between perceived texture qualities and computed visual texture properties. Frontiers in Human Neuroscience, 10, p. 343

Jha, S., Balaji, M.S., Peck, J., Oakley, J. and Deitz, G.D., 2020. The Effects of Environmental Haptic Cues 
on Consumer Perceptions of Retailer Warmth and Competence. Journal of Retailing.

Jonauskaite, D., Wicker, J., Mohr, C., Dael, N., Havelka, J., Papadatou-Pastou, M., Zhang, M. and Oberfeld, D., 2019. A machine learning approach to quantify the specificity of colour-emotion associations and their cultural differences. Royal Society Open Science, 6(9), p.190741.

Joo, J. and Steinert-Threlkeld, Z.C., 2018. Image as data: Automated visual content analysis for political science. arXiv preprint arXiv: 1810.01544

Kareklas, I., Brunel, F.F. and Coulter, R.A., 2014. Judgment is not color blind: The impact of automatic color preference on product and advertising preferences. Journal of Consumer Psychology, 24(1), pp.87-95.

Labrecque, L.I. and Milne, G.R., 2012. Exciting red and competent blue: the importance of color in marketing. Journal of the Academy of Marketing Science, 40(5), pp.711-727.

Lee, A.Y. and Labroo, A.A., 2004. The effect of conceptual and perceptual fluency on brand evaluation. Journal of Marketing Research, 41(2), pp.151-165.

Löfstedt, T., Brynolfsson, P., Asklund, T., Nyholm, T. and Garpebring, A., 2019. Gray-level invariant Haralick texture features. PloS One, 14(2), p.e0212110.

Lou, J., Ren, M. and Wang, H., 2014. Regional principal color based saliency detection. PloS One, 9(11), p.e112475.

Lyons, K., 2017. \#mysanfrancisco: Social Media and the Conceptual Linguistic Landscape. SocArXiv preprint. Retrieved from: https://osf.io/preprints/socarxiv/rqktw/

Matz, S.C., Segalin, C., Stillwell, D., Müller, S.R. and Bos, M.W., 2019. Predicting the personal appeal of marketing images using computational methods. Journal of Consumer Psychology, 29(3), pp.370-390.

McDuff, D. and Berger, J., 2019. Do Facial Expressions Predict Ad Sharing? A Large-Scale Observational Study. arXiv preprint arXiv:1912.10311.

Peck, J. and Childers, T.L., 2003. To have and to hold: The influence of haptic information on product judgments. Journal of Marketing, 67(2), pp.35-48.

Pieters, R., Wedel, M. and Zhang, J., 2007. Optimal feature advertising design under competitive clutter. Management Science, 53(11), pp.1815-1828.

Pieters, R., Wedel, M. and Batra, R., 2010. The stopping power of advertising: Measures and effects of visual complexity. Journal of Marketing, $74(5)$, pp.48-60.

Pridmore, R.W., 2009. Chroma, chromatic luminance, and luminous reflectance. Part I: Basic research and illustration of relations. Color Research Application, 34(1), pp.45-54.

Rosenholtz, R., Li, Y. and Nakano, L., 2007. Measuring visual clutter. Journal of Vision, 7(2), pp.17-17.

Sample, K.L., Hagtvedt, H. and Brasel, S.A., 2019. Components of visual perception in marketing contexts: a conceptual framework and review. Journal of the Academy of Marketing Science, pp.1-17.

Schwarz, M.W., Cowan, W.B. and Beatty, J.C., 1987. An experimental comparison of RGB, YIQ, LAB, HSV, and opponent color models. ACM Transactions on Graphics (TOG), 6(2), pp.123-158.

Shapiro, S., 1999. When an ad's influence is beyond our conscious control: Perceptual and conceptual fluency effects caused by incidental ad exposure. Journal of Consumer Research, 26(1), pp.16-36.

Sievert, C. 2019. 'plotly' R package. [Link]

Stamps III, A.E., 2002. Entropy, visual diversity, and preference. The Journal of General Psychology, 129(3), pp.300320.

Steinert-Threlkeld, Z.C., 2019. The Future of Event Data Is Images. Sociological Methodology, 49(1), pp.68-75.

Townsend, C. and Kahn, B.E., 2014. The "visual preference heuristic": The influence of visual versus verbal depiction on assortment processing, perceived variety, and choice overload. Journal of Consumer Research, 40(5), pp.993-1015

Witzel, C., O'Regan, J.K. and Hansmann-Roth, S., 2017. The dress and individual differences in the perception of surface properties. Vision Research, 141, pp.76-94.

Xi, N., Ma, D., Liou, M., Steinert-Threlkeld, Z.C., Anastasopoulos, J. and Joo, J., 2020, May. Understanding the Political Ideology of Legislators from Social Media Images. In Proceedings of the International AAAI Conference on Web and Social Media (Vol. 14, pp. 726-737)

Xian, S.X. and Shevell, S.K., 2004. Changes in color appearance caused by perceptual grouping. Visual Neuroscience, 21(3), pp.383-388.

Yantis, S., 2005. How visual salience wins the battle for awareness. Nature Neuroscience, 8(8), pp.975-977

Zumbach, D. (2019) R package 'colorfindr'. [Link]

Zvoleff, A. (2019). R Package 'glcm'. [Link] 